\title{
Aplastic Anemia in Egypt: Current Situation and Future Prospective
}

\author{
Safaa A. A. Khaled \\ Department of Internal Medicine, Hematology \& BMT Unit, Assiut University Hospital, Faculty of Medicine, Assiut University, Assiut, \\ Egypt
}

Email address:

safaakhaled2003@gmail.com,sa_ah_mh@yahoo.com

\section{To cite this article:}

Safaa A.A. Khaled. Aplastic Anemia in Egypt: Current Situation and Future Prospective. American Journal of Internal Medicine. Vol. 4, No. 2, 2016,pp. 36-42. doi: 10.11648/j.ajim.20160402.12

Received: April 6, 2016; Accepted: April 20, 2016; Published: May 11, 2016

\begin{abstract}
Aplastic anemia (AA) is a hypoproliferative anemia that led to a significant morbidity and mortality. Recently, dramatic improvement in prognosis of AA was achieved; this was in part due to advances in immunosuppressive therapy and HSCT. Nevertheless, AA is still one of the most challenging hematological disorders that could face a hematologist. This study was conducted at Assiut University Hospital (AUH), Assiut, Egypt to evaluate current situation and future prospective of AA. 63 patients with AA were prospectively enrolled in the study; they were admitted at the Hematology unit, AUH in the period ${ }^{1 \text { sttan }} 2011$ to ${ }^{\mathrm{Feb}} 2012$, a 38 patients with iron deficiency anemia (IDA) were included for comparison. Patients' demographic and clinical data were collected through medical history and clinical examination. Both direct and indirect health care costs of anemia were assessed Direct parameters included doctors' and hospital fees, costs of laboratory investigations, medication fees and costs of HSCT or any procedure. Indirect costs included travelling expenses and earning losses. Social burden of anemia was estimated by anemia related morbidity and mortality. Data were analyzed with SPSSV.17, results showed that AA affected young age group with mean age $30.89 \pm 13.39$ years, without sex predilection, acquired AA was more common than idiopathic (55.6\% vs. $44.4 \%)$, however mortality was higher in idiopathic AA (46.2\%). Causes of aplasia were exposure to chemicals, drugs and hepatitis in order. Only $63.5 \%$ of AA were admitted once and $47.6 \%$ in general ward, the mean hospital days for patients with AA was $17.73 \pm 11.10$, compared to $8.32 \pm 6.69$ in IDA, $\mathrm{P}=0.000$, also higher socio-economic burden of AA was found compared with IDA. These results denoted that management of AA is still ineffective, and that there is a definite need for a strict plan to prevent incidence of AA, particularly in developing countries where effective management of AA is too expensive and relatively unavailable.
\end{abstract}

Keywords: Aplastic Anemia, Future, Perspectives

\section{Introduction}

Although identified as a benign hematological disorder, AA is a devastating disease with consequences and outcomes similar to malignant disorders. Clinical course of AA was found dependent on the degree of aplasia, in severe forms of the disease rapid deterioration of patient's condition and possible incidence of fatal complications is the role. Fortunately, the clinical course of non-severe AA varies from one patient to another, many patients may remain stable for years, others may deteriorate in very short time. Meanwhile clinical course and outcome of acquired AA is similar to that of idiopathic disease, the historical differences between idiopathic and acquired AA is practically not existed $[1,2]$.
AA was found more prevalent in developing than developed countries, and its incidence was linked to low socioeconomic status [3-5].

Until now there are many modalities for treatment of AA; however HSCT is the definite treatment. [6-8] In Egypt, HSCT is only available for a small proportion of patients; [9] furthermore the overall health services provided to patients with AA are very little compared to those in developed countries. This is mainly due to the low socioeconomic status of the country, besides lack of public awareness of AA.

Most published research on AA focused on diagnosis and management of the disease; however the consequences and burden of AA was not investigated in many countries. To our knowledge this is the first research that investigated effects 
and consequences of AA including the economic and social influences of AA. The main aim was to analyze current situation and burden of AA and to recommend how to deal with the disease from its roots rather than to manage its consequences. Another goal was to raise the attention of physicians and hematologists about risk factors for AA in their community. The last objective was to warn authorities in Egypt about AA, and to demonstrate the importance of considering AA a serious but preventable disease as hepatitis.

\section{Materials and Methods}

\subsection{Study Design and Subjects}

This study was conducted at Hematology unit of Internal Medicine Department at AUH. AUH is a big tertiary health care center at Assiut Governorate Egypt; it offers super speciality health services to people of Upper Egypt. Many difficult, complicated and rare cases are referred to AUH from nearby Governorates and health care centers. Patients with AA who were admitted at Hematology and BMT unit in the period 1stJan 2011 to Feb 2012 were prospectively enrolled in the study; also those with IDA who were admitted in the same period were included as a control group. Willingness and agreement for participation in the study were important inclusion criteria.

\subsection{Methods}

\subsubsection{Data Collection}

Data on admission were collected for each patient in the study. These included demographic, social, clinical and hematologic data. These data were collected through medical history taking, thorough clinical examination and laboratory investigations. Questions to verify the possible underlying etiology for AA were addressed during patients' interview such as history of drug intake (e.g. chloramphenicol, busulfan), environmental exposure to toxic agents (e.g benzene exposure) and hepatitis infection (e.g history of fever or jaundice). Patients with idiopathic or acquired AA were enrolled in the study. They were subjected to strict daily follow up strategy throughout their stay in the hospital. During follow up treatment plans, emergence of any disease complications or drug side effects were recorded. After discharge patients came for follow up at hematology outpatient clinics some of them were presented at the emergency room and were re-admitted again. They were also subjected to follow up during each admission episode.

\subsubsection{Diagnosis of Anemia in the Study Groups}

Diagnosis of AA was based on both clinical suspicion and laboratory certainty with peripheral hemogram and bone marrow aspirate or biopsy. Patients were identified to have severe AA if BM cellularity $<25 \%$ plus two or more of the following 1) absolute neutrophil count (ANC) $<0.5 \times 109 / \mathrm{L}, 2)$ reticulocyte count $<20 \times 109 / \mathrm{L}, 3)$ platelet count $<20 \times$ $109 / \mathrm{L}$, if the $\mathrm{ANC}<0.2 \times 109 / \mathrm{L}$ very severe AA was diagnosed. Those with peripheral hemogram that did not meet the criteria of severe AA with hypocellular bone marrow were categorized as non-severe AA. [10] IDA was diagnosed with signs and symptoms suggestive of anemia, microcytic hypochromic anemia in $\mathrm{CBC}$ and blood film, reduced serum iron and raised total iron binding capacity (TIBC).

\subsubsection{Treatment of the Study Groups}

Patients were admitted at hematology unit, AUH those with severe or very severe AA were admitted at the hematology intensive care unit. According to published guidelines for treatment of AA, AA was managed with supportive treatment in the form of transfusions to correct anemia and thrombocytopenia, broad spectrum parenteral antibiotics and antifungals as indicated. However growth factors and erythropoietin were administered to selected cases with acquired AA. Meanwhile over transfusions were avoided to safeguard against alloimmunization particularly in those prone to HSCT. Arrangements for HSCT were done in those with available matched donors, otherwise patients received immunosuppressive therapy. $[11,12]$

IDA was managed by treatment of the underlying etiology combined with oral iron and vitamin $\mathrm{C}$ supplementation. Parenteral iron was administered to those with severe anemia or intolerance to oral iron. Packed RBCs transfusion was done in selected cases to safeguard against anemic heart failure.

\subsubsection{Assessment of Socio- Economic Burden of Anemia in the Study Groups}

Economic burden was assessed quantitatively; indicators for economic burden were categorized as direct and indirect health care costs. The former included diagnosis, admission and treatment expenses while the later comprised loss of income due to lack of productivity or sick leave and travelling expenses. The economic burden was calculated at individual level by estimating the proportion of the direct and indirect costs of the household income.

Social burden of disease was estimated by close follow up of patients during each admission period to detect anemia related morbidity and mortality.

Socio-economic burden of anemia was estimated during the period of hospital admission excluding costs of outpatient follow up and costs before admission; however socioeconomic burden for those who were readmitted during the study period was the sum of burden for all admissions.

\subsubsection{Ethical Considerations}

The study objectives, design and methodology were consistent with the World Medical Association (WMA) declaration of Helsinki for ethics in medical research. [13] Research protocol and objectives were discussed to all the study participants before enrollment in the study. Both patients' oral and written consent for participation in the study were obtained from the study subjects. Furthermore all patients knew well that they can withdraw from the study at any time without affecting their medical care.

\subsubsection{Statistical Analysis}

Data were collected then introduced into a personal 
computer substituting patients' names with code numbers. Analysis of the collected data was done with SPSS V. 17 software (SPSS Inc. Chicago, TL, USA). The mean \pm SD, median, and range were used to describe quantitative variables while qualitative variables were expressed as percentages from the total number. The independent sample T-test was used to compare means while the chi-square test was used to analyze differences among qualitative variables.

\section{Results}

\subsection{Characteristics of the Study Population}

\subsubsection{Demographic Characteristics of the Study Population}

A total of 101 patients were prospectively enrolled in the study, among them 63 were with AA and 38 with IDA, their ages ranged from 18-80 years old. The mean age of the total group was 31.01 , and $30.89 \& 31.21$ for those with AA and IDA, respectively. There was no sex predilection in AA while IDA was commoner in females of the study groups and the female to male ratio was 1:1 for AA and 1.1:1 for IDA. The vast majority of patients were from rural community for both AA and IDA $(66.7 \% \& 68.4 \%$, respectively). Analysis of geographic distribution of patients showed that most patients were from Assiut governorate where AUH existed (65.3\%). The vast majority of patients with AA were parents (55.6\%) while those with IDA were singles $(47.4 \%)$, and table 1 , showed demographic characteristics of the study groups.

Table 1. Demographic characteristics of the study groups (total $n=101$ ).

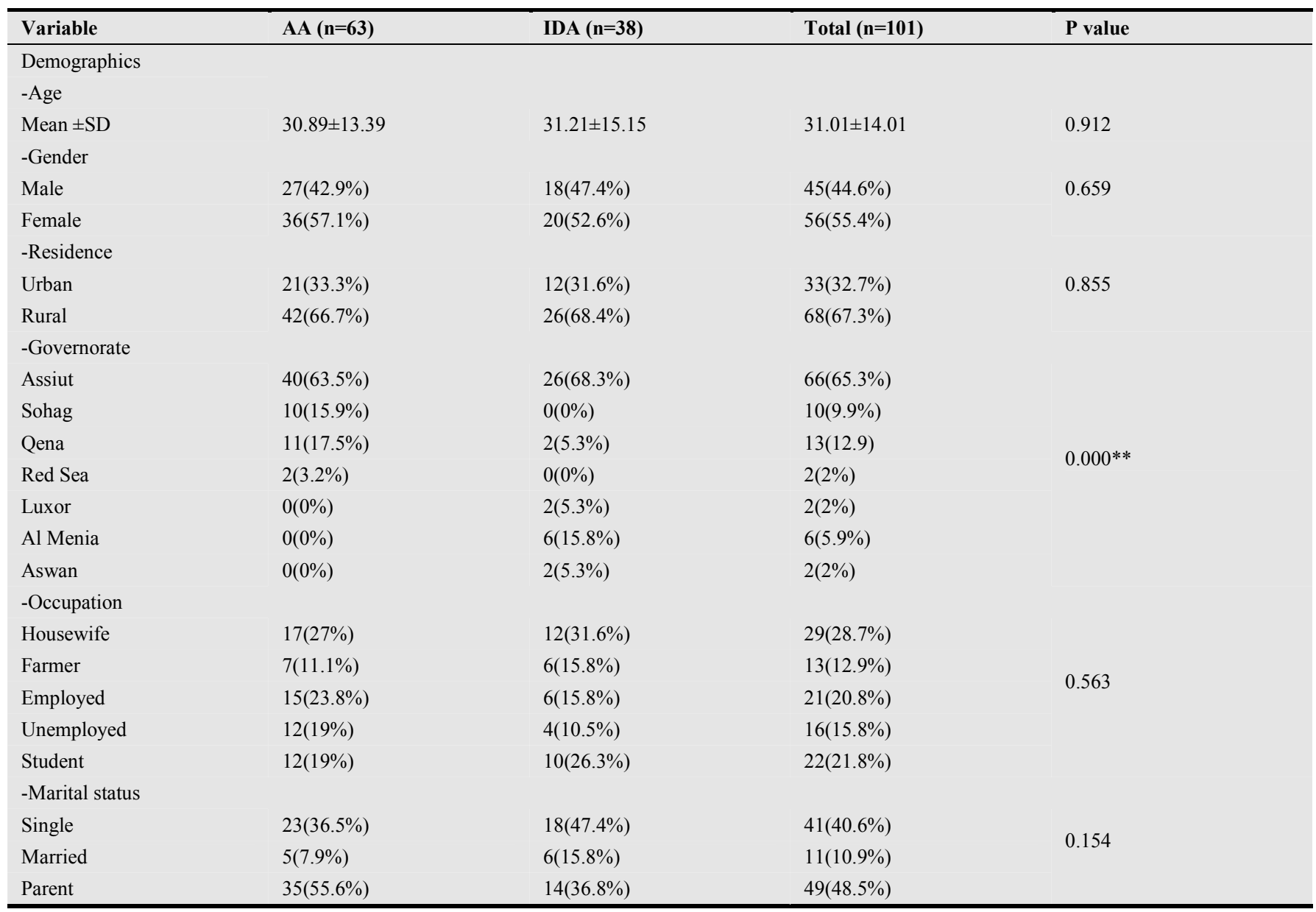

N.B.AA $=$ Aplastic anemia, $\mathrm{IDA}=$ Iron deficiency anemia. Data were presented as mean $\pm \mathrm{SD}$, or as percentage from the total number as appropriate.

\subsubsection{Clinical, Hematologic and Disease Characteristics of the Study Participants}

The most common presenting complaints of patients with AA were symptoms of anemia, thrombocytopenia and lastly leucopenia while the most prominent sign was purpuric eruptions (65.1\%). Expectedly leucopenia, anemia, macrocytosis and thrombocytopenia were the $\mathrm{CBC}$ findings of AA patients compared with IDA where normal WBCs, anemia, microcytosis and thrombocytosis were predominant findings $(\mathrm{P}=0.000,0.07,0.000 \& 0.000$, respectively $)$.

Acquired AA was the most common type of AA in this study $(55.6 \%$ Vs $44.4 \%)$ for idiopathic AA. The most common causes of aplasia were exposure to chemicals, drug induced, and post-hepatitic $(23.8 \%, 19.1 \%$ \& $12.7 \%$, respectively). Severity of AA among the study group were severe, very severe and non severe categories (39.7\%, 20.6\% $\$ 39.7 \%$ respectively), and table 2 , showed clinical and hematologic characteristics of the study groups. 
Table 2. Clinical and hematologic characteristics of the study groups (total $n=101$ ).

\begin{tabular}{lllll}
\hline Variable & AA $(\mathbf{n}=\mathbf{6 3})$ & IDA $(\mathbf{n}=\mathbf{3 8})$ & Total $(\mathbf{n}=\mathbf{1 0 1})$ & P value \\
\hline Main complaint & & & & \\
-Pallor, dizziness & $30(47.6 \%)$ & $38(100 \%)$ & $68(67.3 \%)$ & $0.000^{* *}$ \\
-Bleeding tendency & $16(25.4 \%)$ & $0 \%$ & $16(15.8 \%)$ & \\
-Fever & $17(27 \%)$ & $0 \%$ & $53(52.5 \%)$ & $0.000^{* *}$ \\
Signs & & & $41(40.6 \%)$ & $0.000^{* *}$ \\
-Pallor & $15(23.8 \%)$ & $38(100 \%)$ & 0.073 \\
-Purpura & $41(65.1 \%)$ & $0(0 \%)$ & $0.000^{* *}$ \\
-Signs of infection & $7(11.1 \%)$ & $0(0 \%)$ & $31.1 \pm 2.65$ & $0.000^{* *}$ \\
C.B.C & & & $8.3 \pm 10.57$ & $0.000^{* *}$ \\
-WBCs & $3.9 \pm 2.41$ & $6.04 \pm 2.5$ & $81.3 \pm 14.64$ & \\
-Hb & $6.5 \pm 1.90$ & $10.71 \pm 16.92$ & $1.2 \pm 0.83$ & \\
-MCV & $90.2 \pm 6.55$ & $66.5 \pm 12.06$ & & \\
-Plt & $45.9 \pm 50.59$ & $5.04 \mathrm{E} 2 \pm 210.372$ & \\
-Retic. & $1.8 \pm 0.99$ & $0.89 \pm 0.45$ & & \\
Type of AA & & & \\
-Idiopathic & $28(44.4 \%)$ & & & \\
-Acquired & $35(55.6 \%)$ & & & \\
Severity of AA & & & \\
-Severe & $25(39.7 \%)$ & & \\
-Very severe & $13(20.6 \%)$ & & \\
Non-severe & $25(39.7 \%)$ & & \\
Causes of acquired AA & & & \\
-Post-hepatitis & $8(12.7 \%)$ & $12(19.1 \%)$ & & \\
-Drug induced & $15(23.8 \%)$ & & \\
-Chemical exposure & & & \\
\hline
\end{tabular}

N.B.AA $=$ Aplastic anemia, $I D A=$ Iron deficiency anemia. $\mathrm{WBCs}=$ white blood cells, $\mathrm{Hb}=$ hemoglobin, $=$ mean corpuscular volume, Plt $=$ platelet, $\mathrm{Retic}=$ reticulocyte count. Data were presented as mean $\pm \mathrm{SD}$, or as percentage from the total number as appropriate.

Table 3. Economic burden of anemia in the study groups (total no=101).

\begin{tabular}{|c|c|c|c|}
\hline EconomicVariable & $\mathrm{AA}(n=63)$ & $\operatorname{IDA}(n=38)$ & P value \\
\hline \multicolumn{4}{|l|}{ Medical ensurance } \\
\hline -Ensured & $25(39.7 \%)$ & $2(5.3 \%)$ & \multirow[t]{2}{*}{$0.000 * *$} \\
\hline -Un-ensured & $38(60.3 \%)$ & $36(94.7 \%)$ & \\
\hline \multicolumn{4}{|l|}{ Admission place } \\
\hline -General ward & $30(47.6)$ & $38(100 \%)$ & \multirow[t]{2}{*}{$0.000 * *$} \\
\hline Intensive care unit & $33(52.4 \%)$ & $0(0 \%)$ & \\
\hline \multicolumn{4}{|l|}{ Frequency of admission } \\
\hline -Twice & $19(30.2 \%)$ & $0(0 \%)$ & \multirow{2}{*}{$0.000 * *$} \\
\hline -Thrice & $4(6.3 \%)$ & $0(0 \%)$ & \\
\hline Hospital days & $17.73 \pm 11.10$ & $8.32 \pm 6.69$ & $0.000 * *$ \\
\hline \multicolumn{4}{|l|}{ Direct costs } \\
\hline -Doctor's fees & $1.01 \mathrm{E} 2 \pm 112.70$ & $68.94 \pm 64.33$ & 0.066 \\
\hline -Admission fees & $3.64 \mathrm{E} 3 \pm 2504.41$ & $1.43 \mathrm{E} 3 \pm 878.08$ & $0.000 * *$ \\
\hline Costs of laboratory tests- & $6.08 \mathrm{E} 2 \pm 399.27$ & $1.92 \mathrm{E} 2 \pm 95.59$ & $0.000 * *$ \\
\hline -Transfusion Fees & $6.92 \mathrm{E} 2 \pm 725.58$ & $10.53 \pm 31.10$ & $0.000 * *$ \\
\hline -Procedure fees & $2.08 \mathrm{E} 4 \pm 48347.17$ & $36.64 \pm 108.85$ & $0.000 * *$ \\
\hline \multicolumn{4}{|l|}{ Indirect costs } \\
\hline -Travelling expenses & $1.22 \mathrm{E} 2 \pm 88.89$ & $1.02 \mathrm{E} 2 \pm 99.74$ & 0.1 \\
\hline -Earning losses & $4.95 \mathrm{E} 2 \pm 737.71$ & $2.11 \mathrm{E} 2 \pm 289.08$ & \\
\hline Monthly family income- & $3.29 \mathrm{E} 3 \pm 705.15$ & $3.25 \mathrm{E} 3 \pm 540.84$ & 0.185 \\
\hline -Total direct costs & $2.81 \mathrm{E} 4 \pm 47761.71$ & $2.05 \mathrm{E} 3 \pm 838.11$ & $0.000 * *$ \\
\hline -Total Indirect costs & $6.17 \mathrm{E} 2 \pm 753.25$ & $3.12 \mathrm{E} 2 \pm 330.98$ & $0.001 *$ \\
\hline
\end{tabular}

N.B. $A A=$ Aplastic anemia, IDA= Iron deficiency anemia. All costs were calculated in LE.

\subsection{Economic Costs of Aplastic Anemia}

The frequency of admission for patients with AA throughout their follow up period was once, twice, and thrice $(63.5 \%, 30.2 \%$ and $6.3 \%)$ respectively, while $100 \%$ of IDA were admitted once at the general ward. Only $47.6 \%$ of patients with AA were admitted at the general ward and $52.4 \%$ in the hematology intensive care unit. The mean duration of hospital days for AA was 17.73 compared to 8.32 for IDA, $(P=0.000)$, and hospital charges were 3.64E3 LE for patients with AAvs. 1.43E3 LE for those with IDA, $(\mathrm{P}=$ 0.000 ). The mean monthly income of patients with AA was 
3.29E3 LEvs. 3.25E3 LE for IDA, $\mathrm{P}=0.18$. The mean direct and indirect health care expenses for patients with AA were 2.81E4 \& 6.17E2 LEvs. 2.05E3\&3.12E2 LE for those with IDA, $\mathrm{P}=0.001 \& 0.02$ respectively. The expenditure on direct health care was 8 times the monthly family income in patients with AA, while that for IDA comprised $63.6 \%$ of family income. The expenditure on indirect health care was $18.8 \%$ of family income for AA vs. $9.3 \%$ for IDA, and table 3 , showed economic burden of anemia in the study groups.

\subsection{Social Burden of Aplastic Anemia}

Social burden of AA was assessed by estimating AA

$\begin{array}{lll}\text { Gender } & \begin{array}{l}\text { Governorate } \\ \text { Assiut:61.5\% }\end{array} & \text { Social } \\ \text { Male:53.8\% } & \text { Sohag:7.7\% } & \text { Urban:46.2\% } \\ \text { Female:46.2\% } & \text { Quena:30.8\% } & \text { Rural:53.8\% } \\ & \text { RedSea:0\% } & \end{array}$

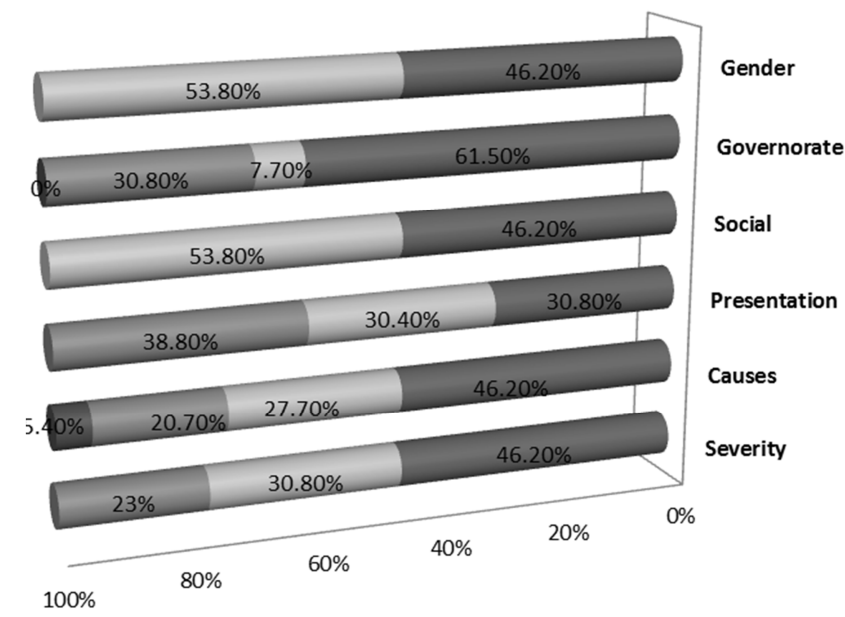

Figure 1. Factors affecting mortality in patients with aplastic anemia.

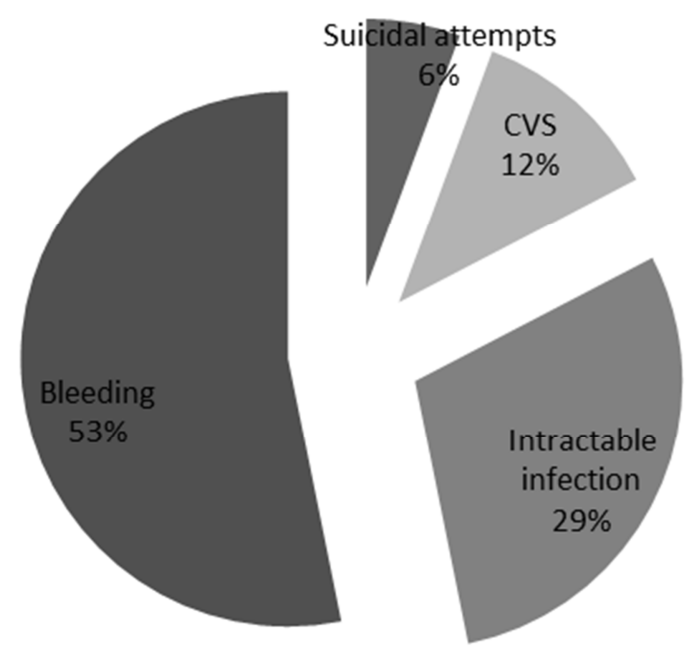

Figure 2. Aplastic anemia related morbidity. CVS= cereberovascular stroke.

$56.5 \%$ of patients with AA showed various morbidities, the most common were bleeding, intractable infections, cerebrovascular stroke and suicidal attempts in descending order, and figure 2 showed aplastic anemia related morbidity. related mortality and morbidity in the study group.In this study $20.6 \%$ of patients with AA passed their way during their hospital stay, while there were no reported deaths among the group of IDA. Nearly, $69.2 \%$ of deaths due to AA were parents. AA related mortality was higher in elderly patients, males (53.8\%), disease severity $(76.8 \%$ in severe and very severe cases), cause of aplasia (50.7\% in idiopathic aplasia), type of treatment $(60.3 \%$ received supportive measures only), and low socio-economic class $(55.7 \%$ from rural community), figure 1 showed factors affecting mortality in patients with AA.
Presentation

Bleeding:30.8\%

Infection: $30.4 \%$

Anemia:38.8\%
Causes

Idiopathic: $46.2 \%$

Chemicals: $27.7 \%$

Drugs: $20.7 \%$

Posthepatitic: $5.4 \%$
Severity

Verysevere: $46.2 \%$

Severe: $30.8 \%$

Non-severe: $23 \%$

\section{Discussion}

Aplastic anemia was reported as a rare disease; however these reports were based on western registry data. [14] Furthermore it is assumable that incidence of AA will be different from one geographic region to another due to the well known association between AA and environmental, genetic, occupational and infectious patterns from one region to another. A study in India showed that nearly 6,000 cases diagnosed with AA per year. [5] A study by Mahmoud et al. showed that AA comprised $21 \%$ of a total of 1300 patients who received HSCT over 18-years in Egypt. [9]

The current research studied current situation and future prospectives of AA, in Egypt. In this study the median age of patients with AA was obviously lower than that in other studies (30-years vs. 53 \&40-years), meanwhile it was consistent with other studies. Unlike other studies where biphasic age distribution was noted for AA the peak age for AA in this study were late teens. $[5,15,16]$

In this study anemia AA showed no sex predilection, on the other hand IDA was more prevalent in females than males; these findings were albeit consistent with others who found that anemia in general is commoner in girls and young aged females. Meanwhile these results were contradictory to other researchers who considered AA to be a disease of young males. In this study both AA and IDA were related to low socio-economic status, these findings confirmed the previously reported association of low socio-economic status with anemia. [17-19] Why AA is related to socio-economic status? This could be explained by the extensive exposure to environmental and infectious factors that could predispose to AA.

The majority of patients with AA were presented with symptoms suggestive of anemia and thrombocytopenia, this presentation was similar to other studies. [2, 3]

Acquired AA was more prevalent than idiopathic AA in the study group this was inconsistent with other studies, [10] however this difference could be explained by the higher age 
range of patients of the current study; at these ages idiopathic AA is unlikely. On the other hand these findings were nearly similar to the findings of Ibrahiem et al. who found that acquired AA comprised $57.5 \%$ of AA patients who were admitted at AUH, hematology unit in the period from Nov2011 to Oct2012. [20]

Although allogeniec HSCT is the only curative treatment for AA, it was available for only small proportion of patients in this study. This may be due to high expenses of HSCT and unavailability of matched sibling donors for most of the study group. In Egypt, HSCT was found to cost 15,000 USD all of it is provided by health insurance and Ministry of health. [9] Lack of trained health professionals and limited transplant centers were other reasons for rarity of HSCT in Egypt. Furthermore the relatively effective antithymocyte globulin was not available for our patients with AA as it is too expensive. [21]

In this study a higher proportion of patients with AA, compared with other studies, presented with severe and very severe form of disease, and passed their way during the period of hospital stay. $[7,15,16]$ This could be explained by many factors including late presentation of patients as they were referred from far governorates, patients' ignorance, insufficient health care facilities, and unavailability of the most effective therapies for AA, HSCT and immunosuppressive therapy for most of our patients

Although the cost of HSCT in Egypt is much lower than that in developed countries it was available for only a small proportion of our patients. [22-25] This may be due to many reasons including low socio-economic status of the country, lack of proper insurance for most of the patients, uneven distribution of transplantation centers all over the republic, where it is mainly in Cairo which is hardly accessible for many patients in Upper Egypt. Furthermore, lack of stem cell registry in Egypt makes the availability of unrelated matching donor very difficult.

The expenditure on direct and indirect health care services of AA was obviously higher than that of IDA; meanwhile there was no difference in family income of the patients. This could be explained that patients with AA and IDA were from the same socio-economic class; however patients with AA were admitted for longer periods besides expenses of intensive care services, HSCT and higher earning losses. In Egypt, patients received health services at university/government hospitals free of charge or with minimal fee, nevertheless, the expenditure on indirect health care services was paid by either the patients or their families. Our results reflected findings of most researchers that the highest burden of AA is expenses of HSCT. [22-26]

\section{Conclusion}

In conclusion AA anemia is a disease of poorer classes of population who could not offer out of pocket money for proper management of this potentially fatal disease. Furthermore, AA is still a disease with very poor prognosis in Egypt. Also this study showed a profound economic and social impact of AA whether on a population or an individual level. On the light of this study several strategies are still needed to reduce the socioeconomic burden of AA. Firstly urgent preventive protocols are needed to combat not only AA but other endemic infectious diseases such as viral hepatitis that led to AA. Next it is very crucial to commence national stem cell donor registry in Egypt, and to encourage people to donate stem cells to help patients find matched donors. Also there is a definite need for construction of transplant centers capable of performing allogeniec HSCT in other governorates other than Cairo, to provide services to people of Upper Egypt. Furthermore it is very important to cut short the referral and administrative measures for HSCT to guard against transplant failure due to the unavoidable multiple transfusions and save unnecessary costs during the waiting period. Finally a nationwide health education programs are required to increase public awareness regarding AA its causes, prevention, treatment and possible complications.

Although HSCT is the most expensive therapeutic option for AA, however it is highly cost effective option. Accordingly we recommend that patients with matched donors should undergo HSCT early in the disease to save costs of hospital admission, investigations, transfusions, and other fees.

\section{Acknowledgements}

The author wish to express deep thanks and gratitude to Prof. Esam El-Bieh the head of hematology unit for his marvelous help and support

\section{References}

[1] Smith E G, Marsh JC. Acquired aplastic anemia, other acquired bone marrow failure disorder and dyserythropoiesis. In: Hoffbrand AV, Catovsky D, Tudenham E, editors. Postgraduate Haematology. 5th ed. Oxford: Blackwell publishing Ltd; 2005: 965-78.

[2] Brodsky RA, Jones. Aplastic Anemia. The Lancet London, 2005.7-13; 365 (9471): 1647-57.

[3] Young NS. Acquired aplastic anemia; in Schechter GP, Berliner N, Telen MJ (eds): Hematology. Washington, The American Society of Hematology Education Program Book, 2000, pp 18-38.

[4] Adil SN, Burney JA, Kakepto GN, Khurshid M. Epidemiologic features of Aplastic Anemia in Pakistan. J Pak Med Assoc. 2001; 51 (12): 443-5.

[5] Malhotra P, GellaV, Murthy GSG, Varma N, and Varma S. Higdh incidence of aplastic anemia is linked with lower socioeconomic status of Indian population. J Public Health 2015;doi:10.1093/pubmed/fdv027.

[6] Fuhrer M, Ramp fU, Baumann I, et al. Immunosuppressive therapy for aplastic anemia in children: a more severe disease predicts better survival. Blood 2005; 15 (6): 2102-4.

[7] Ali AS, Hamid MH, Khan MA, Ahmed FN. Acquired Aplastic Anemia- and experience with different drug therapies. Pak Paediat J2003; 27 (1): 19-27. 
[8] Hernandez-Rivera EG. Hematopoietic stem-cell transplantation in aplastic anemia. Rev Invest Clini 2005; 57 (2): 298-304.

[9] Mahmoud HK, El-Haddad AM, Fahmy OA, et al. Hematopoietic stem cell transplantation in Egypt. Bone Marrow Transplantation (2008) 42, S76-S80.

[10] Davies JK, Guinan EC. An update on the management of severe idiopathic aplastic anemia in children. Br J Haem.2007; 136 (4): 549-564.

[11] Young NS, Calado R, Scheinberg P. Current concepts in the pathophysiology and treatment of aplastic anemia. Blood 2006; 108: 2509-19.

[12] Tichelli A, Schrezenmeier H, Bacigalupo A. Immunosuppressive treatment of aplastic anemia: Schrezenmeier H, Bacigalupo A., editors. Aplastic anemia: Pathophysiology and treatment. Cambridge UK: Cambridge University press, 2000; 154-96.

[13] $64^{\text {th }}$ World Medical Association General Assembly, Fortaleza, Brazil 2013. All rights reserved. http://www.wma.net/en/30publications/10policies/b3

[14] Dallman PR, Johnson C. Prevalence and causes of anemia in the United States, 1976 to1980. AmJ Clin Nutr1984; 39: 43745.

[15] Mary JY, Baumelou M, Guiguet M. The French Cooperative Group for Epidemiological Study of Aplastic Anemia. Epidemiology of aplastic anemia in France: a prospective multicentric study. Blood 1990; 75: 1646-53.

[16] Montané E, Ibáñez L, Vidal X, et al. Haematologica. Epidemiology of aplastic anemia: aprospective multicenter study. 2008 Apr; 93 (4): 518-23.

[17] Clausen N, Salmi T, Storm Mathisen I, Johannesson G. Severe
Aplastic Anemia in Nordic Countries: A population based study of incidenc, course and outcome. Archives Dis Childhood 1996; 74: 319-22.

[18] Kim JY, Shin S, HanK, et al. Relationship between socioeconomic status and anemia prevalence in adolescent girls based on the fourth and fifth Korea National Health and Nutrition Examination Surveys. Eur J Clin Nutr. 2014 Feb; 68 (2): 253-8.

[19] Issaragrisil S, Kaufman DW, Anderson TE, et al. An association of aplastic anaemia in Thailand with low socioeconomics tatus. Br J Haematol. 1995 Sep; 91 (1): 80-4.

[20] Ibrahiem OA, Haridi MA, Kamel RA. Outcomes of aplastic anemia patients in Assiut University hospital, one year study. 2014. Assiut Med. J.; 38 (1): 247-254.

[21] Field SR, Follmann D, Nunez O, Neal S, Young MD. ATG and Cyclosporin-A for severe aplastic anemia. JAMA 2003; 289 (9): 1130-35.

[22] Khera N, Zeliadt SB, Lee SJ. Economics of hematopoietic cell transplantation. Blood. 2012; 8: 1545-51.

[23] Lee SJ, Klar N, Weeks JC, et al. Predicting costs of stem-cell transplantation. JClinOncol.2000; 18: 64-71.

[24] Saito AM, Cutler C, Zahrieh D, et al. Costs of allogeneic hematopoietic cell transplantation with high dose regimens. Biol Blood MarrowT ransplant. 2008; 14: 197-207.

[25] Espérou H, Brunot A, Roudot-Thoraval F, et al. Predicting the costs of allogeneic sibling stem-cell transplantation: results from a prospective, multicenter, Frenchstudy. Transplantation. 2004; 77: 1854-58.

[26] Sharma SK, Choudhary D, Gupta N, et al. Cost of Hematopoietic Stem Cell Transplantation in India. Mediterr J Hematol Infect Dis. 2014; 6 (1): e2014046. 